Pisoni. Speech Perception in Deaf Children

\title{
SPEECH PERCEPTION IN DEAF CHILDREN WITH COCHLEAR IMPLANTS
}

\author{
David B. Pisoni \\ Dept. of Psychology, Indiana University, Bloomington, Indiana, USA \\ pisoni@indiana.edu
}

\begin{abstract}
Cochlear implants work reasonably well in many profoundly deaf adults and children. For a prelingually deaf child, the electrical stimulation transmitted by a cochlear implant represents the introduction of a new sensory modality that provides spectral and temporal information about speech and spoken language. Despite the success of cochlear implants in many deaf children, large individual differences have been reported on a wide range of speech and language outcome measures. This finding is observed in all research centers around the world. Some children do extremely well with their cochlear implant while others derive only minimal benefits after receiving their implant. Understanding the reasons for the variability in outcomes and the large individual differences following cochlear implantation is one of the most important problems in the field today. In this paper, I present a brief summary of recent findings on the speech perception skills of deaf children following cochlear implantation. The results of these studies suggest that in addition to several demographic variables, variation in children's success with cochlear implants also reflects fundamental differences in rapid phonological coding and verbal rehearsal processes used in working memory.
\end{abstract}

\section{INTRODUCTION}

A cochlear implant $(\mathrm{Cl})$ is a surgically implanted electronic device that functions as an auditory prosthesis for patients with severe to profound sensorineural hearing losses. The device provides electrical stimulation to the surviving spiral ganglion cells of the auditory nerve while bypassing the damaged hair cells of the inner ear to restore hearing. A cochlear implant provides both adults and children with access to sound and sensory information from the auditory modality. All of the cochlear implants in use today consist of an internal multiple electrode array and an external processing unit. The external unit consists of a microphone that picks up sound energy from the environment and a signal processor that codes frequency, amplitude and time and compresses the signal to match the narrow dynamic range of the ear. Cochlear implants provide temporal and amplitude information. Depending on the manufacturer, several different place coding techniques are used to represent and transmit frequency information in the signal. Since the approval of cochlear implants by the FDA as a treatment for profound deafness, over 60,000 patients have received cochlear implants at centers all over the world (Clarke, 2003).

For a postlingually deaf adult with a profound hearing loss, a cochlear implant provides a transformed electrical signal to an already fully developed auditory system and intact mature language processing system. These patients have already acquired spoken language under normal listening conditions so we know their central auditory system and brain are functioning normally. For a congenitally deaf child, however, a cochlear implant provides novel electrical stimulation through the auditory sensory modality and an opportunity to perceive speech sounds and develop spoken language for the first time after a period of auditory deprivation. 
Pisoni. Speech Perception in Deaf Children

Congenitally deaf children have not been exposed to speech and do not develop spoken language normally. Although their brain and nervous system continue to develop in the absence of normal auditory stimulation, recent findings suggest that some cortical reorganization has already taken place during the period of sensory deprivation before implantation and that several aspects of speech and language skills after implantation may develop in an atypical fashion. Both peripheral and central differences in neural function are likely to be responsible for the wide range of variability observed in outcome and benefit following implantation.

For the last 12 years, I have been studying congenitally deaf children who have received cochlear implants. These children are the most interesting and theoretically important clinical population to study because they have been deprived of sound and auditory stimulation at a very early point in neural and cognitive development. After implantation, their hearing is restored with electrical stimulation that is designed to simulate the response of a healthy cochlea to speech and other auditory signals. Aside from the obvious clinical benefits of cochlear implantation as a method of treating profound prelingual deafness in children, this clinical population also provides a unique opportunity to study the effects of auditory deprivation on the development of speech perception and language processing skills and to assess the effects of restoration of hearing via artificial electrical stimulation of the nervous system.

In some sense, one can think of research on this clinical population as the modern-day analog of the so-called "forbidden experiment" in the field of language acquisition, except that in this case after a period of sensory deprivation has occurred, hearing is restored via medical intervention and children begin to receive exposure to sound and stimulation through the auditory modality. Under these conditions, it is possible to study both the consequences of a period of auditory deprivation on speech and language development as well as the effects of restoring hearing using artificial electrical stimulation of the auditory nerve.

While cochlear implants work well for many profoundly deaf children, they do not always provide benefits to all children who receive them. Numerous studies have shown that the variation in audiological outcomes and benefits following cochlear implantation is enormous. Some children do extremely well with their cochlear implants and display near-typical speech perception and language skills on a wide range of traditional clinical speech and language tests when tested under quiet listening conditions in the laboratory. In contrast, other children struggle for long periods of time after they receive their cochlear implant and often never achieve comparable levels of speech and language performance or verbal fluency.

Why are some children doing so well with their Cls while others struggle and perform more poorly? How can we explain these differences in outcome and benefit? What underlying factor or set of factors are responsible for these large differences in performance on a wide range of behavioral tests? If we can identify the reasons why a good child is performing so well, we should be able to use this basic knowledge to help poorer performing children improve their speech and language skills and achieve their potential to derive optimal/maximal benefit from their Cls.

What do we know about outcome and benefit in deaf children with Cls? Table I lists seven key findings observed universally at all implant centers around the world. The findings indicate that several demographic and medical factors contribute to outcome and benefit following 
Pisoni. Speech Perception in Deaf Children

implantation. In addition to the enormous variability observed in the speech and language outcome measures, several other findings have been consistently reported in the clinical literature on cochlear implants in deaf children. An examination of these findings provides some preliminary insights into the possible underlying cognitive and neural basis for the variability in outcome and benefit among deaf children with cochlear implants. When these contributing factors are considered together, it is possible to begin formulating some specific hypotheses about the reasons for the enormous variability in outcome and benefit.

Table I. Seven Key Findings on Cochlear Implants in Deaf Children
1. Large Individual Differences
2. Age of Implantation (Sensitive Periods)
3. Effects of Early Experience (Oral vs. TC)
4. Cross-Modal Plasticity
5. Links Between Perception \& Production
6. No Preimplant Predictors of Outcome
7. Abilities Emerge after Implantation (Learning)

Almost all of the clinical research on cochlear implants has focused on the effects of a small number of demographic variables using traditional outcome measures based on assessment tools developed by clinical audiologists and speech pathologists. Although rarely discussed explicitly in the literature, these behaviorally-based clinical outcome measures of performance are the final product of a large number of complex sensory, perceptual, cognitive and linguistic processes that contribute to the observed variation among cochlear implant users. Until recently, little if any research focused on the underlying information processing mechanisms used to perceive and produce spoken language in this clinical population. Our investigations of these fundamental neurocognitive and linguistic processes have provided some new insights into the basis of individual differences in profoundly deaf children with cochlear implants.

Age at implantation is another factor that has been shown to influence all outcome measures of performance. Children who receive an implant at a young age do much better on a whole range of outcome measures than children who are implanted at an older age (Kirk, 2000). Length of auditory deprivation or length of deafness is also related to outcome and benefit. Children who have been deaf for shorter periods of time before implantation do much better on a variety of clinical measures than children who have been deaf for longer periods of time. Both findings demonstrate the contribution of sensitive periods in sensory, perceptual and linguistic development and serve to emphasize the close links between neural development and behavior, especially the development of hearing, speech and language (Konishi, 1985; Marler \& Peters, 1988).

Early sensory and linguistic experience and language processing activities after implantation have also been shown to affect performance on a wide range of outcome measures. Implanted children who are immersed in Oral-only communication environments do much better on clinical tests of speech and language development than implanted children who are enrolled in Total Communication programs (Kirk, Pisoni, \& Miyamoto, 2000). Oral communication approaches emphasize the use of speech and hearing skills and actively encourage children to produce 
Pisoni. Speech Perception in Deaf Children

spoken language to achieve optimal benefit from their implants. In contrast, total communication approaches employ the simultaneous use of some form of manual-coded English along with speech to help the child acquire language using both sign and spoken language inputs.

Until just recently, clinicians and researchers have been unable to find reliable preimplant predictors of outcome and success with a cochlear implant (see, however, Bergeson \& Pisoni, 2004). The absence of preimplant predictors is a theoretically significant finding because it suggests that many complex interactions take place between the newly acquired sensory capabilities of a child after a period of auditory deprivation, properties of the language-learning environment and various interactions with parents and caregivers that the child is exposed to after receiving a cochlear implant. More importantly, however, the lack of preimplant predictors of outcome and benefit makes it difficult for clinicians to identify those children who are doing poorly with their cochlear implant at a time in development when changes can be made to modify and improve their language processing skills.

Finally, when all of the outcome and demographic measures are considered together, the available evidence strongly suggests that the underlying sensory and perceptual abilities for speech and language "emerge" after implantation. Performance with a cochlear implant improves over time for almost all children. Success with a cochlear implant therefore appears to be due, in part, to perceptual learning and exposure to a language model in the environment (Clarke, 2003). Because outcome and benefit with a cochlear implant cannot be predicted reliably from traditional behavioral measures obtained before implantation, any improvements in performance observed after implantation must be due to sensory and cognitive processes that are linked to maturational changes in neural and cognitive development (see Sharma, Dorman \& Spahr, 2002).

Although these demographic factors can account for a large portion of the variance in outcomes, there are still substantial gaps in our basic knowledge of how Cls work in the brain. Moreover, several other sources of variability related to the "information processing" capacities of the children have also been found to contribute to outcome. These factors involve the sensory and perceptual encoding of speech, the storage and processing of phonological and lexical information in short-term memory and response output. There are also several linguistic factors that reflect the use of common phonological representations in a range of behavioral tasks that are routinely used to assess speech and language outcome and measure benefit following cochlear implantation.

One of the primary research questions deals with the nature of the variation in outcome and the reasons for the large individual differences in effectiveness of Cls. If we can structure, shape, modify and reorganize how the central auditory, cognitive and linguistic processes are working in these children, perhaps we can provide more benefit to the low-performing children and explain the observed variability in outcomes.

We are not only interested in whether deaf children with Cls are able to "hear" via their $\mathrm{Cl}$ (i.e., detection and discrimination). We are also interested in what they are able to do with the sensory information they do hear. How do they go from continuous waveforms to discrete words? How do these children construct phonological representations of the input signals they hear? What kinds of linguistic representations do they create? Are their phonological and lexical 
Pisoni. Speech Perception in Deaf Children

representations "fully specified" like normal-hearing typically developing children or are they "underspecified" reflecting more primitive coarse-coding strategies and the use of broader phonetic categories? What can deaf children with $\mathrm{Cls}$ do with these representations? How are these representations used in speech perception, word recognition and spoken language comprehension? How are they used in speech production? And how are they used in other linguistic tasks like sentence comprehension, language production, and reading?

To investigate individual differences and the sources of variation in outcome, we began by analyzing a set of data from a longitudinal project on cochlear implants in children (see Pisoni et al., 1997; 2000). Our first study examined the exceptionally good users of cochlear implantsthe so-called Stars. These are the children who did extremely well with their cochlear implants after only two years of implant use. The Stars are able to acquire spoken language quickly and easily and appear to be on a developmental trajectory that parallels normal-hearing children although delayed a little in time (see Svirsky et al., 2000). The theoretical motivation for studying the exceptionally good children was based on an extensive body of research on "expertise" and "expert systems" theory (Ericsson \& Smith, 1991). Many important new insights have come from studying expert chess players, radiologists, spectrogram readers, like Victor Zue, and other individuals who have highly developed domain-specific skills.

\section{ANALYSIS OF THE Stars}

Using an extreme groups design, we initially sorted a large sample of deaf children who used Cls $(\mathrm{N}=160)$ into two categories based on their PBK phoneme scores after two years of implant use. The PBK test is an open-set test of spoken word recognition that requires the child to repeat back isolated English words spoken by an examiner using live-voice presentation (Haskins, 1949). The PBK test is considered to be very difficult for most deaf children who have received Cls and is considered to be the "gold standard" of outcome performance. The Stars consisted of children who scored in the top 20 percent of the distribution on the PBK test; the low-performers were the children in the bottom 20 percent of the distribution. We then examined the children's performance on several different speech and language outcome measures over a six year period (see Pisoni, et al., 1997; 2000). In this section, we describe the results of the speech feature perception and spoken word recognition tests obtained from this study. Other findings are reported in our earlier papers.

Speech Feature Perception. Measures of speech feature perception for consonants and vowels were obtained for both groups of subjects with the Minimal Pairs Test (Robbins et al., 1988). This test uses a two-alternative forced-choice picture pointing procedure. The child hears a single word spoken in isolation on each trial by the examiner using live-voice presentation and is required to select the picture that corresponds to the test word.

A summary of the consonant perception results for both groups of children is shown in Figure 1. Percent correct perception is displayed separately for manner, voicing and place of articulation as a function of implant use in years. Data for the Stars are shown by the filled bars; data for the low performers are shown by the open bars. Chance performance on this task is $50 \%$ correct as shown by the solid line. A second horizontal line is also shown in this figure at $70 \%$ correct corresponding to scores that are significantly above chance using the binominal distribution. 
Pisoni. Speech Perception in Deaf Children

\section{Speech Feature Perception}

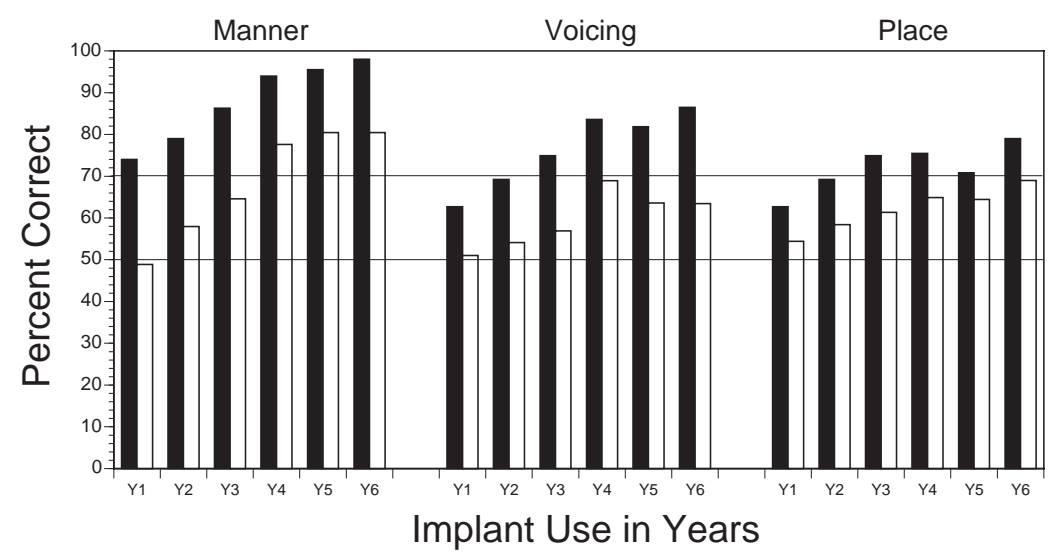

Figure 1. Percent correct recognition on the Minimal Pairs Test (MPT) for manner, voicing and place as a function of implant use. The Stars are shown by filled bars, the low performers are shown by open bars.

Inspection of the results for the minimal pairs test obtained over a period of six years of implant use reveals several consistent findings. First, performance by the Stars was consistently better than performance by the low performers for every comparison across all three consonant features. Second, perception improved over time with implant use for both groups overall, although the increases were primarily due to improvements in perception of manner and voicing by the Stars. At no interval did the mean scores of the low performers exceed chance performance on perception of voicing and place features. Although increases in performance were observed over time for this group, their perception scores never reached the levels of performance observed with the Stars, even for the gross manner contrasts that eventually exceeded chance performance in years 4,5 and 6 .

The results of the minimal pairs test indicate that both groups of children have difficulty perceiving fine phonetic details of isolated spoken words even in a two-choice closed-set testing format. The Stars were able to discriminate differences in manner of articulation after one year of implant use and they showed consistent improvements in performance over time for both manner and voicing contrasts but they still had more difficulty reliably discriminating differences in place of articulation, even after five years of implant use. In contrast, the low performers were just barely able to discriminate differences in manner of articulation after four years of implant use and they were unable to reliably perceive differences in voicing and place of articulation even after five or six years of use.

The pattern of results shown in Figure 1 suggests that both groups of children are encoding spoken words using "coarse" phonetic representations that contain much less fine-grained phonetic detail than normal hearing children typically use. Their lexical representations appear to be "underspecified" compared to the representations that normal-hearing children typically use. The Stars are able to reliably discriminate manner and to some extent voicing much earlier after implantation than the low performing children. They also display consistent improvements in speech feature perception over time. These speech feature perception skills are assumed to 
Pisoni. Speech Perception in Deaf Children

place initial constraints on the basic sensory information that can be used for subsequent word learning and lexical development. It is very likely that if a child cannot reliably perceive differences between pairs of spoken words that are acoustically similar under these relatively easy forced-choice test conditions, he/she will also have a great deal of difficulty recognizing words in isolation with no context or retrieving the phonological representations of these sound patterns from memory for use in simple speech production tasks such as imitation or immediate repetition. We would also expect these children to have a great deal of difficulty in recognizing and imitating nonwords that have no lexical representations.

Open-Set Word Recognition. Two word recognition tests, the Lexical Neighborhood Test (LNT) and the Multi-syllabic Lexical Neighborhood test (MLNT), were used to measure open-set word recognition skills in both groups of subjects (Kirk, Pisoni \& Osberger, 1995). Both tests use words that are familiar to normal-hearing preschool age children. The LNT contains monosyllabic words, the MLNT contains polysyllabic words. Each test uses two different sets of words in order to measure lexical discrimination and provide details about how the lexical selection process is carried out. Half of the items in each test consist of lexically "easy" words and half consist of lexically "hard" words. The differences in performance on these two types of items in each test provide an index of how well a child is able to make fine phonetic discriminations among phonetically similar words. Differences in performance between the LNT and the MLNT provide a measure of the extent to which the child is able to make use of word length cues to recognize and access words from the mental lexicon. The items on both tests were presented in isolation one at a time by the examiner using live voice auditory-only presentation. A mesh screen was used to cover the talker's face. The child was required to repeat back the test item immediately after it was presented by the examiner on each trial.

Figure 2 shows percent correct word recognition on the LNT and the MLNT for the Stars as a function of years of implant use. Scores for the "easy" and "hard" words are shown separately for each test by year. Within each year of use, the results for the LNT are shown on the left while the MLNT results are shown on the right. Several important differences are shown here that provide some insight into the task demands and processing operations used in open-set word recognition tests. First, the Stars consistently demonstrated higher levels of word recognition performance on both the LNT and the MLNT than the low performers. These differences were present across all six years but they are most prominent during the first three years after implantation. Word recognition scores for the low performers on both tests were low and close to the floor. Normal-hearing children typically display ceiling levels of performance on both of these tests by age 4 (see Kluck et al., 1997).

Two other theoretically important findings are also shown in this figure. First, the Stars displayed evidence of a word length effect at each testing interval. Recognition was always better for the long words than short words. This pattern was obscured by a floor effect for the low performers who were unable to do this open-set task at all during the first three years. The presence of a word length effect for the Stars demonstrates that they recognize words "relationally" in the context of other words that they have in their lexicon (Luce \& Pisoni, 1998). If these children were simply recognizing words in isolation, feature-by-feature or segment-by-segment, without reference to other words they already know and can access from their lexicons, we would expect that performance would be worse for longer words than shorter words because longer words contain more information. The pattern of findings shown in Figure 2 is exactly the 
Pisoni. Speech Perception in Deaf Children

opposite of this prediction and parallels earlier results obtained with normal-hearing adults and children (Luce \& Pisoni, 1998; Kirk et al., 1995; Kluck et al., 1997). Longer words are easier to recognize than shorter words because they are more distinctive and discriminable and therefore less confusable with other phonetically similar words. The present findings suggest that the Stars are recognizing words based on their knowledge of other words in the language and generalizations about sublexical patterns of words in their lexicons using processing strategies that are similar to those used by normal-hearing listeners.

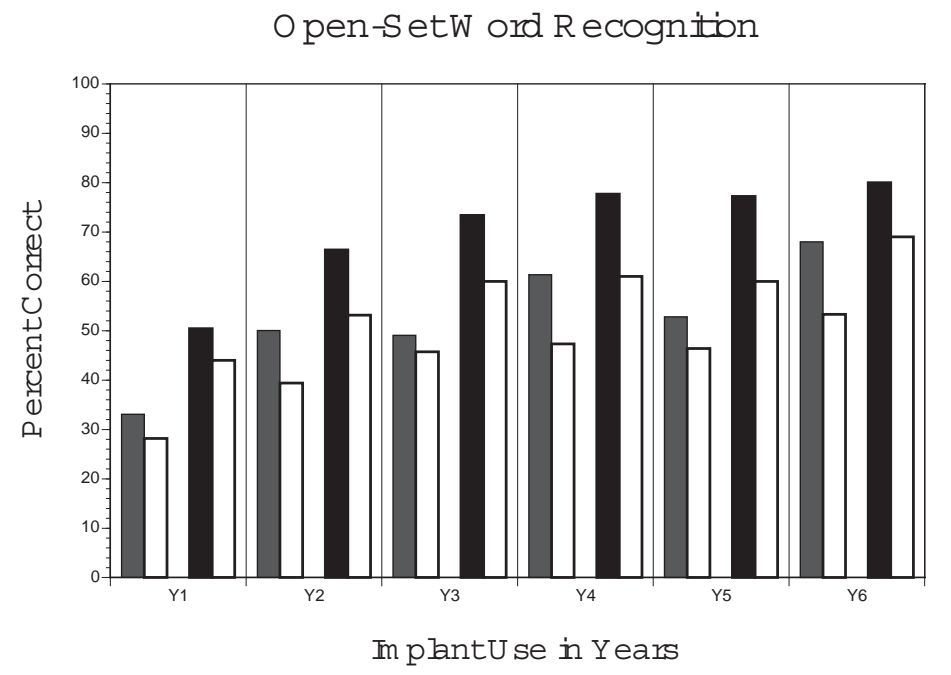

Figure 2. Percent correct word recognition performance of the Stars on the Lexical Neighborhood Test (LNT) and the Multisyllabic Lexical Neighborhood Test (MLNT) as a function of implant use and lexical difficulty. "Easy Words" are shown by filled bars, "Hard Words" are shown by open bars.

Additional support for the role of the lexicon and the use of lexical knowledge of words in openset word recognition is provided by a second finding shown in this figure. The Stars also showed a consistent effect of "lexical discrimination" for the words on both tests. They recognized lexically "easy" words better than lexically "hard" words according to predictions of the NAM model of spoken word recognition (see Luce \& Pisoni, 1998). The NAM model assumes that words are organized and stored in an acoustic-phonetic similarity space in long-term lexical memory and that words are recognized in the context of other phonetically similar words by processes of bottom-up acoustic-phonetic activation followed by top-down lexical competition. The difference in performance between "easy" words and "hard" words is present for both tests but it is larger and more consistent over time for the words on the MLNT test. The low performers did not show the same pattern of sensitivity to lexical competition among the test words.

The differences in performance observed between these two groups of children on both openset word recognition tests are not at all surprising and were anticipated because these two extreme groups were initially created based on their PBK scores, another open-set word recognition test. The overall pattern of the results is theoretically important because the findings obtained with these two open-set word recognition tests demonstrate that the skills and abilities 
Pisoni. Speech Perception in Deaf Children

used to recognize isolated spoken words are not specific to the words used on the PBK test. The initial differences between the two groups were replicated with two other open-set word recognition tests using different sets of words.

Correlations Among Outcome Measures. We also found that the speech feature perception and spoken word recognition scores from the Stars along with several other outcome measures such as sentence comprehension, vocabulary knowledge, expressive and receptive language and speech intelligibility were all highly inter-correlated with each other (Pisoni et al., 2001). This pattern of results suggests the existence of a common underlying source of variance. We have suggested that this source of variance is related to the rapid construction of phonological representations in speech perception and the use of a set of common phonological processing skills across a wide range of different behavioral tests that are routinely used to measure outcome and assess benefit following cochlear implantation.

The speech perception and word recognition data obtained from these children were based on traditional audiological outcome measures that were collected as part of their annual clinical assessments. The scores on these tests are "endpoint measures" of the child's behavior that reflect the final product of a long series of cognitive, perceptual, and linguistic analyses of the input signal. Process measures of performance that assess what a child does with the sensory information provided by his/her cochlear implant were not obtained in the standard research protocol used in our longitudinal study of the Stars so it was impossible to measure information processing capacity, processing speed or learning. To determine if fundamental differences in immediate memory capacity, processing speed and learning might be responsible for the variation in performance, we obtained several new process measures of performance on another group of children with Cls.

\section{NEW PROCESS MEASURES OF PERFORMANCE}

Immediate Memory Capacity. The information processing capacity of immediate memory was measured using forward and backward WISC digit spans in a large group ( $\mathrm{N}=176)$ of 8- and 9year old children who had used their cochlear implants for at least five years (see Pisoni \& Cleary, 2003). We found that their forward digit spans which are assumed to reflect early phonological coding and verbal rehearsal skills were "atypical" compared to a group of agematched normal-hearing children $(\mathrm{N}=44)$. Both forward and backward digit spans were shorter for children with $\mathrm{Cls}$ than normal-hearing children suggesting limitations on processing capacity of immediate memory. We also found that children who were enrolled in oral communication (OC) programs had longer forward digit spans than children enrolled in total communication (TC) programs. This result demonstrates significant effects of early linguistic experience and activity-dependent learning on information processing capacity of immediate memory.

Verbal Rehearsal Speed. Estimates of the deaf children's verbal rehearsal speed were also obtained in this study by measuring speaking rates from spoken sentences based on sentence durations (see Pisoni \& Cleary, 2003). The results of this analysis revealed slower verbal rehearsal speeds. As shown in other typical-developing populations, verbal rehearsal speed was also found to be strongly correlated with immediate memory capacity (i.e., forward digit spans) as well as several independent measures of spoken word recognition in isolation and sentence context and a measure of speech intelligibility (see Pisoni \& Cleary, 2004). 
Pisoni. Speech Perception in Deaf Children

Scanning of STM. Several novel measures of speech timing during the digit span recall task were also used to investigate retrieval and scanning of phonological information in STM and speed of articulation at output (see Burkholder \& Pisoni, 2003). We found that scanning of familiar digits was significantly slower for the children with Cls, suggesting less robust perceptual encoding and active maintenance of phonological representations of digits in STM. In addition, we found that early linguistic experience also affected the scanning rates. Children who were immersed in oral (OC) educational programs displayed faster scanning rates for items in STM than children who were in total communication (TC) programs.

Sequence Memory Spans. Using a novel experimental methodology, based on MiltonBradley's Simon memory game, we also measured reproductive memory spans for auditory (A), visual (V) and auditory + visual (AV) sequences (see Cleary, Pisoni \& Geers, 2001; Pisoni \& Cleary, 2004). Sequences of spoken color names (A) or colored lights (V) or color names combined simultaneously with colored lights $(A+V)$ were presented randomly on each trial using the Simon game box. Subjects were asked to reproduce the sequence by pressing the appropriate colored panels on the Simon box. With an adaptative testing algorithm, we obtained several measures of the longest sequence a subject could correct reproduce. Across all three presentation conditions, we found that deaf children with Cls displayed shorter reproductive memory spans than normal-hearing children and adults. More interestingly, they also showed smaller redundancy gains in the $\mathrm{A}+\mathrm{V}$ multimodal presentation condition compared to $\mathrm{A}$-only and $\mathrm{V}$-only unimodal presentation conditions. Also, as reported in the memory literature, the normalhearing children and adults both showed a "modality effect," that is, they had longer memory spans for auditory sequences of color names compared to visual sequences of colored lights. In contrast, the deaf children with Cls displayed a reversal of the "modality effect" found with the normal-hearing children and adults. Their memory spans for visual sequences spans were actually longer than their spans for auditory sequences suggesting differential effects of crossmodal plasticity and reorganization as a consequence of a period of deafness early in life before implantation.

Sequence Learning Spans. The Simon memory game methodology was also used to study simple sequence learning and repetition effects under the same three presentation conditions used in the memory experiments (see Pisoni \& Cleary, 2004). In the learning experiment, if a sequence was reproduced correctly on a given trial, the same sequence was repeated again on the next trial but the length of the sequence was increased by one item chosen randomly from the set of four colors. We found that the sequence learning spans were much shorter for children with Cls than children with normal hearing across all three presentation conditions. More importantly, about one third of the deaf children with Cls failed to show any sequence repetition effects or learning at all. This result was observed across all three presentation conditions indicating the presence of significant differences in simple sequence learning based on pattern repetition that are not modality specific in nature. These findings suggest that a period of sensory deprivation due to deafness at early stages of development produces effects on the central nervous system and the cortical areas that are involved in learning, memory and cognitive processes used to encode and maintain sensory inputs from both visual and auditory modalities. 
Pisoni. Speech Perception in Deaf Children

\section{PHONOLOGICAL DECOMPOSITION AND SUBLEXICAL KNOWLEDGE}

Nonword Repetition Task. To understand the linguistic factors that are responsible for the variation in speech and language outcome measures following implantation, we have examined the use of sublexical phonological knowledge in speech perception with data obtained from a nonword repetition task (Cleary, Dillon et al., 2002; Carter et al., 2002; Dillion et al., 2004a, b). In this task, children are asked to listen to a novel nonsense word that conforms to English phonology and immediately repeat it back to the experimenter. The children are told in advance that the stimuli will be unfamiliar "funny-sounding" words and they should just try to say back whatever they hear on each trial to the best of their ability. Their verbal responses were recorded on DAT tape for later linguistic analysis and playback. Although nonword repetition appears at first glance to be a simple information processing task, in actuality it is a very complex linguistic task that requires the child to perform well on each of the individual component processes including: speech perception, phonological encoding and decomposition, active verbal rehearsal in working memory, retrieval and phonological reassembly and finally phonetic implementation and speech production. One motivation for studying the nonword repetition skills of children with Cls was the assumption in the clinical literature that hearingimpaired children display great difficulty in open-set tests of word recognition because they do not know the meanings of the stimulus words that are used on the test (see Kirk et al., 1995). A second reason was that nonword repetition performance by normal-hearing children has been found to be strongly related to variation in vocabulary development and other language learning milestones (Gathercole \& Baddeley, 1993; Gathercole et al., 1999).

Linguistic Analysis and Perceptual Ratings of Nonword Responses. Twenty nonword patterns were presented to a large group of 8- and 9-year old deaf children $(\mathrm{N}=88)$ with cochlear implants (see Dillon et al., 2004a, b). Responses were then phonetically transcribed by two trained linguists. The nonword responses were also played back to normal-hearing listeners who were asked to make perceptual similarity ratings of each response following presentation of the original nonword pattern. Several scores were computed for each child to measure correct repetition of the consonants and vowels in each nonword stimulus as well as perceptual ratings. These scores were then correlated with several independent measures of performance on the individual component skills. A summary of the correlations of these nonword repetition scores with open-set word recognition (LNT and MLNT), Forward Digit Span, Speech Intelligibility, Speaking Rate, Word Attack, and Rhyme Errors is given in Table II. The Word Attack and Rhyme Errors were obtained from visual reading tasks that were specifically designed to measure single word reading. As shown here, the transcription scores for both consonants and vowels as well as the perceptual ratings were all strongly correlated with these separate component measures. The pattern and consistency of these correlations suggests the use of common phonological representations and analysis skills across a wide range of language processing tasks. The results obtained with the nonword repetition task also suggest an independent and autonomous sublexical level of phonological analysis that is separate and distinct from the lexical level. Like normal-hearing children, deaf children with cochlear implants are able to reproduce and imitate novel word-like patterns. Many of these children were able to decompose unfamiliar nonsense words into smaller linguistically significant segments and to then rapidly retrieve and reassemble these segments into motor outputs for speech production without relying on lexical representations in long-term memory. 
Pisoni. Speech Perception in Deaf Children

Table II. Partial correlations between nonword repetition scores and several speech and language outcome measures (controlling for performance IQ, age at onset of deafness and communication mode)

\begin{tabular}{|l|c|c|c|}
\hline & $\begin{array}{c}\text { Percent Correct } \\
\text { Consonants } \\
(\mathbf{N}=76)\end{array}$ & $\begin{array}{c}\text { Percent Correct } \\
\text { Vowels } \\
(\mathbf{N}=76)\end{array}$ & $\begin{array}{c}\text { Mean Perceptual } \\
\text { Accuracy Rating } \\
(\mathbf{N}=76)\end{array}$ \\
\hline LNT easy words & $+.83^{\star \star \star}$ & $+.78^{\star \star \star}$ & $+.76^{\star \star \star}$ \\
\hline LNT hard words & $+.85^{\star \star \star}$ & $+.71^{\star \star \star}$ & $+.70^{\star \star \star}$ \\
\hline MLNT & $+.77^{\star \star \star}$ & $+.74^{\star \star \star}$ & $+.77^{\star \star \star}$ \\
\hline Forward Digit Span & $+.60^{\star \star}$ & $+.62^{\star \star}$ & $+.76^{\star \star \star}$ \\
\hline Speech Intelligibility & $+.91^{\star \star \star}$ & $+.88^{\star \star \star}$ & $+.87^{\star \star \star}$ \\
\hline Speaking Rate & $-.84^{\star \star \star}$ & $-.81^{\star \star \star}$ & $-.85^{\star \star \star}$ \\
\hline Word Attack & $+.75^{\star \star \star}$ & $+.72^{\star \star \star}$ & $+.78^{\star \star \star}$ \\
\hline Rhyme Errors & $-.63^{\star \star}$ & $-.68^{\star \star}$ & $-.54^{\star}$ \\
\hline
\end{tabular}

${ }^{\star} p<.05,{ }^{\star \star} p<.01,{ }^{\star \star \star} p<.001$

\section{DISCUSSION AND CONCLUSIONS}

What is the common factor that links these diverse sets of findings together? We suggest that the development and efficient use of phonological processing skills is a significant contributor above and beyond the traditional demographic and medical variables that have been shown to affect outcome and benefit following cochlear implantation. Phonological analysis involves the rapid encoding and decomposition of speech into sequences of discrete meaningless phonetic segments or "particles" and the assignment of structural descriptions to these sound patterns that reflect the linguistically significant sound contrasts of the words in the ambient target language. For many years, both clinicians and researchers have considered open-set tests of spoken word recognition performance to be the "gold standard" of outcome and benefit in both children and adults who have received Cls. The reason open-set tests have achieved this privileged status in the field is because they load heavily on several component processes including speech perception, verbal rehearsal and maintenance, retrieval of phonological representations from STM, and phonetic implementation strategies required for speech production, motor control and response output. All of these subprocesses rely on highly automatized phonological processing skills involving analysis and decomposition of the input signal into familiar linguistic units, like features or segments, and the reassembly and synthesis of these units into sequences of motor commands and gestures for output.

When prelingually deaf children receive a cochlear implant as a treatment for their profound hearing loss, they do not simply have their hearing restored at the auditory periphery. More significantly, after implantation they begin to receive substantial auditory stimulation to specialized areas of their central nervous system that are critical for the development of spoken language and specifically for the development of phonological processing skills that are used to rapidly encode and process speech signals. Our recent findings on speech perception and phonological decomposition in deaf children with cochlear implants suggest that in addition to the traditional demographic and medical variables that are able to predict some proportion of the variance in traditional audiological measures of outcome and benefit, there are several additional sources of variance that reflect the contribution of basic information processing skills 
Pisoni. Speech Perception in Deaf Children

commonly used in a wide range of language processing tasks, specifically those which rely on rapid phonological encoding of speech and verbal rehearsal strategies in working memory. Thus, some proportion of the variability and individual differences in outcome following cochlear implantation is related to central auditory, cognitive and linguistic factors that reflect how the initial sensory information transmitted by the cochlear implant is subsequently encoded and processed and how it is used by the listener in specific behavioral tasks that are routinely used to measure speech and language outcomes and assess benefit.

\section{ACKNOWLEDGEMENTS}

Preparation of the paper was supported by NIH-NIDCD Research Grants R01 DC00111 and R01 DC00064 to Indiana University. I would like to thank Miranda Cleary, Caitlin Dillon and Rose Burkholder for their help and advice on all stages of the research described in this paper and Cynthia Clopper for editorial suggestions and advice. I would also like to thank Richard Miyamoto for extending an invitation to me to join his research program on cochlear implants at the Indiana University School of Medicine at Indianapolis.

\section{REFERENCES}

Bergeson, T. \& Pisoni, D.B. (2004). Audiovisual speech perception in deaf adults and children following cochlear implantation. In Handbook of Multisensory Integration (edited by G. Calvert, C. Spence \& B.E. Stein), Cambridge: MIT Press, 749-772.

Burkholder, R. \& Pisoni, D.B. (2003). Speech timing and working memory in profoundly deaf children after cochlear implantation. Journal of Experimental Child Psychology, 85, 63-88.

Carter, A.K., Dillon, C.M. \& Pisoni, D.B. (2002). Imitation of nonwords by hearing impaired children with cochlear implants: Suprasegmental analyses. Clinical Linguistics \& Phonetics, 16, 619-638.

Clarke, G. (2003). Cochlear Implants: Fundamentals and Applications. New York: SpringerVerlag.

Cleary, M., Dillon, C.M. \& Pisoni, D.B. (2002). Imitation of nonwords by deaf children after cochlear implantation: Preliminary findings. Annals of Otology, Rhinology, \& Laryngology Supplement-Proceedings of the $8^{\text {th }}$ Symposium on Cochlear Implants in Children, 111, 9196.

Cleary, M., Pisoni, D.B. \& Geers, A.E. (2001). Some measures of verbal and spatial working memory in eight- and nine-year-old hearing-impaired children with cochlear implants. Ear \& Hearing, 22, 395-411.

Dillon, C.M., Cleary, M., Pisoni, D.B. \& Carter, A.K. (2004a). Imitation of nonwords by hearingimpaired children with cochlear implants: Segmental analyses. Clinical Linguistics and Phonetics, 18, 39-55.

Dillon, C.M., Pisoni, D.B., Cleary, M., and Carter, A.K. (2004b). Nonword imitation by children with cochlear implants: Consonant analyses. Archives of Otolaryngology -Head \& Neck Surgery, 130, 587-591.

Ericsson, K.A., \& Smith, J. (1991). Toward a General Theory of Expertise: Prospects and Limits. New York, NY: Cambridge University Press.

Gathercole, S.E. \& Baddeley, A.D. (1993). Working Memory and Language. Hillsdale, NJ: Lawrence Erlbaum Associates. 
Pisoni. Speech Perception in Deaf Children

Gathercole, S.E., Service, E., Hitch, G.J., Adams, A.M. \& Martin, A.J. (1999). Phonological short-term memory and vocabulary development: Further evidence on the nature of the relationship. Applied Cognitive Psychology, 13, 65-77.

Haskins, H. (1949). A phonetically balanced test of speech discrimination for children. Unpublished Mater's Thesis, Northwestern University, Evanston, IL.

Kirk, K.I. (2000). Challenges in the clinical investigation of cochlear implant outcomes. In Cochlear Implants: Principles and Practices (edited by J.K. Niparko, K.I. Kirk, N.H. Mellon, A.M. Robbins, B.L. Tucci and B.S. Wilson), Philadelphia, PA; Lippincott, Williams \& Wilkins, 225-259.

Kirk, K.I., Pisoni, D.B., \& Miyamoto, R.T. (2000). Lexical discrimination by children with cochlear implants: Effects of age at implantation and communication mode. In Cochlear Implants (edited by S.B. Waltzman \& N.L. Cohen), New York: Thieme, 252-254.

Kirk, K.I., Pisoni, D.B. \& Osberger, M.J. (1995). Lexical effect on spoken word recognition by pediatric cochlear implant users. Ear \& Hearing, 16, 470-481.

Kluck, M., Pisoni, D.B. \& Kirk, K.I. (1997). Performance of normal-hearing children on open-set speech perception tests. Progress Report on Spoken Language Processing \#21, Indiana University, Department of Psychology, Bloomington, IN.

Konishi, M. (1985). Birdsong: From behavior to neuron. Annual Review of Neuroscience, 8, 125170.

Luce, P.A., \& Pisoni, D.B. (1998). Recognizing spoken words: The neighborhood activation model. Ear \& Hearing, 19, 1-36.

Marler, P., \& Peters, S. (1988). Sensitive periods for song acquisition from tape recordings and live tutors in the swamp sparrow, Melospiza georgiana. Ethology, 77, 76-84.

Pisoni, D.B. \& Cleary, M. (2003). Measures of working memory span and verbal rehearsal speed in deaf children after cochlear implantation. Ear \& Hearing, 24, 106S-120S.

Pisoni, D.B. \& Cleary, M. (2004). Learning, memory and cognitive processes in deaf children following cochlear implantation. In Handbook of Auditory Research: Auditory Prosthesis, SHAR Volume X (edited by F.G. Zeng, A.N. Popper \& R.R. Fay), Springer.

Pisoni, D.B., Svirsky, M.A., Kirk, K.I., \& Miyamoto, R.T. (1997). Looking at the Stars: A first report on the intercorrelations among measures of speech perception, intelligibility, and language development in pediatric cochlear implant users. Progress Report on Spoken Language Processing \#21, Indiana University, Department of Psychology, Bloomington, IN.

Pisoni, D.B. (2000). Cognitive factors and cochlear implants: Some thoughts on perception, learning, and memory in speech perception. Ear \& Hearing, 21, 70-78.

Pisoni, D.B., Cleary, M., Geers, A.E. \& Tobey, E.A. (2000). Individual differences in effectiveness of cochlear implants in prelingually deaf children: Some new process measures of performance. Volta Review, 101, 111-164.

Robbins, A.M., Renshaw, J.J., Miyamoto, R.T., Osberger, M.J., \& Pope, M.L. (1988). Minimal pairs test. Indianapolis, IN: Indiana University School of Medicine.

Sharma, A., Dorman, M.F. \& Spahr, A.J. (2002). A sensitive period for the development of the central auditory system in children with cochlear implants: Implications for age of implantation. Ear \& Hearing, 23, 532-539. 
Pisoni. Speech Perception in Deaf Children

Svirsky, M.A., Robbins, A.M., Kirk, K.I., Pisoni, D.B. \& Miyamoto, R.T. (2000). Language development in profoundly deaf children with cochlear implants. Psychological Science, 11, 153-158. 